\title{
Sabot front borerider stiffness vs. dispersion: Finding the knee in the curve ${ }^{1}$
}

\author{
Alan F. Hathaway and John R. Burnett, Jr. \\ Arrow Tech Associates, 1233 Shelburne Road, Suite \\ D8, Pierson House, South Burlington, Vermont 05403, \\ USA \\ E-mail: arrowtech@prodas.com
}

In the design of armor piercing, fin-stabilized, discarding sabot projectiles, the radial stiffness of the sabot front borerider has a significant impact on the projectile's dispersion and is, therefore, an important design consideration. Whether designing a new projectile or trying to improve an existing design, projectile designers can achieve front borerider stiffness without understanding its affect on dispersion characteristics. There is a knee in the stiffness vs. dispersion curve at which a change in the sabot front borerider stiffness will have a significant impact on dispersion or no impact at all depending on whether the stiffness is increased or decreased. The subject of this paper is an analytical approach to quantitatively determine the knee in the curve. Results from using this approach on the M865 APFSDS projectile are also presented.

\section{Introduction}

The ability to predict the dispersion of a projectile is a challenging task due to the fact that dispersion is a combination of independent and interdependent random launch "events" occurring throughout the complete projectile environment from propellant ignition to target impact. There are many parameters that influence each of these "events". One of the more important parameters affecting these "events" is the stiffness of the sabot front borerider.

If the stiffness is insufficient, testing during projectile development might reveal that the dispersion is outside the design specifications; this leads to design changes and additional testing. However, since dispersion is a

\footnotetext{
${ }^{1}$ Presented at the 9th US Army Symposium on Gun Dynamics, McLean, VA, November 17-19, 1998.
}

combination of "events", insufficient stiffness could be masked by other "events" during this phase of testing. It is also unlikely that sufficient testing is performed during development to provide assurance that, across the total range of manufacturing tolerances for every part/assembly, all combinations of these "events" have been tested. During production, insufficient stiffness could mean failing a Target Impact Dispersion (TID) Lot Acceptance Test (LAT) requirement; this type of failure can be very costly.

The in-bore balloting components of dispersion, which are directly affected by sabot bore rider stiffness, can be analytically determined using a worst case scenario in terms of muzzle exit conditions. However, it is often very time consuming and requires many trials to find the specific initial conditions that produce the worst muzzle exit conditions.

This paper summarizes an analytical method that combines in-bore balloting analyses, dispersion sensitivity determination, and manufacturing tolerance information into a stochastic approach for predicting dispersion with varying sabot front borerider stiffness.

\section{Overview of stochastic approach to predicting dispersion}

\subsection{Why stochastic approach}

One of the primary contributors to the in-bore balloting component of dispersion is the accumulation of tolerances in the manufactured projectile parts and assembly of those parts into a projectile whose CG does not lie on the geometric center of the projectile. With high velocity, conventional guns, very small CG offsets can produce catastrophic results. While a user may want to know what the worst possible launch scenario may be, he/she may also be interested in the likelihood of a failure occurring. Use of a stochastic approach enables the analyst to determine the probability of failure (either structural or launch induced dispersion). It also gives the user the range and mean of expected behavior. 
Due to the fact that the relationship between maximum balloting loads and the initial orientation of projectile CG offset and principal axis tilt is not intuitive, selection of a "worst case" analysis condition could require several trial and error analysis replications. An analytical procedure that is set up to only run one set of conditions at a time is of limited value and can prove to be very time consuming. It has been Arrow Tech's experience that an approach that randomly selects initial conditions and continues with a sound analytical solution can prove very useful in determining the probability of occurrence of the outcome.

The question is sometimes asked: "Why do I need a stochastic approach when I only launch one or, at most, a handful of projectiles at a time?"

The answer to this question is, at the same time, both simple and complex. Projectiles launched from high performance gun systems are sensitive to small imperfections. While it is true that high performance projectiles are fabricated with significant precision, the center of mass is not perfectly aligned with the geometric center of the projectile. Assembly of the piece parts and/or manufacture of any projectile is also imperfect (dimensional and runout tolerances). These small differences of the mass axis from perfection can have an orientation with the geometric axis that is not predictable.

Another random source of error is the placement of the projectile within the bore. The projectile (even if perfectly manufactured) will never be positioned exactly the same way within the bore on each successive shot. The small differences in this, and the projectile mass axis offsets, when combined with minor deviations of the bore centerline from a straight gun tube can produce different maximum stress conditions within each projectile firing.

\subsection{Approach considers the complete projectile environment}

This approach considers the complete projectile environment from propellant ignition to target impact as shown in Fig. 1. The environment includes the following three major phases:

1. Propellant ignition to muzzle exit

2. Muzzle exit until sabot discard

3. Sabot discard through target impact

Each of these distinct phases involves parameters that have an impact on dispersion and requires a specific type of analysis to determine the components of dispersion.
Propellant ignition to muzzle exit implies an in-bore balloting analysis is performed to determine the muzzle exit condition components of dispersion. Arrow Tech's BALANS analysis software is used for the balloting analysis. The basic inputs for the in-bore balloting analysis are a lumped parameter model of the projectile that properly characterizes its mass properties and flexibility including the sabot front borerider stiffness, a forcing function, and several diameters and runouts that are used to orient the projectile within the gun tube.

Muzzle exit to sabot discard dispersion components consist of a bore site component and a sabot discard component. These transition parameters are very difficult to determine analytically. For this reason, the bore site component is usually determined via test set-up measurements and the sabot discard component is determined from experience with testing of similar projectiles.

Sabot discard through target impact is the free-flight trajectory of the projectile. The dispersion components are muzzle velocity variation, wind sensitivity, aerodynamics jump and trim angle, and aerodynamic mass asymmetries. Arrow Tech's Projectile Design and Analysis system, PRODAS, is used for determining the sensitivities to these dispersion components.

The dispersion components from the complete projectile environment are then combined in a statistical Target Impact Dispersion (TID) analysis to determine the overall dispersion both horizontally and vertically.

\section{Analysis system for determining dispersion vs. stiffness}

\subsection{Input data requirements}

The process begins with gathering basic technical information such as manufacturing and assembly drawings and/or specifications, analytical results from other analyses such as a finite element analysis of the sabot front borerider. This information is critical to building the accurate analytical model of the projectile to be used during all analyses within this approach. From this information, a tolerance study can also be performed for input into the in-bore balloting analysis.

The second piece of information required is production history information such as Statistical Process Control (SPC) information. Even if working with a new projectile design for which there is no production history, it is valuable to obtain this information for a similar design or a projectile with similar characteris- 


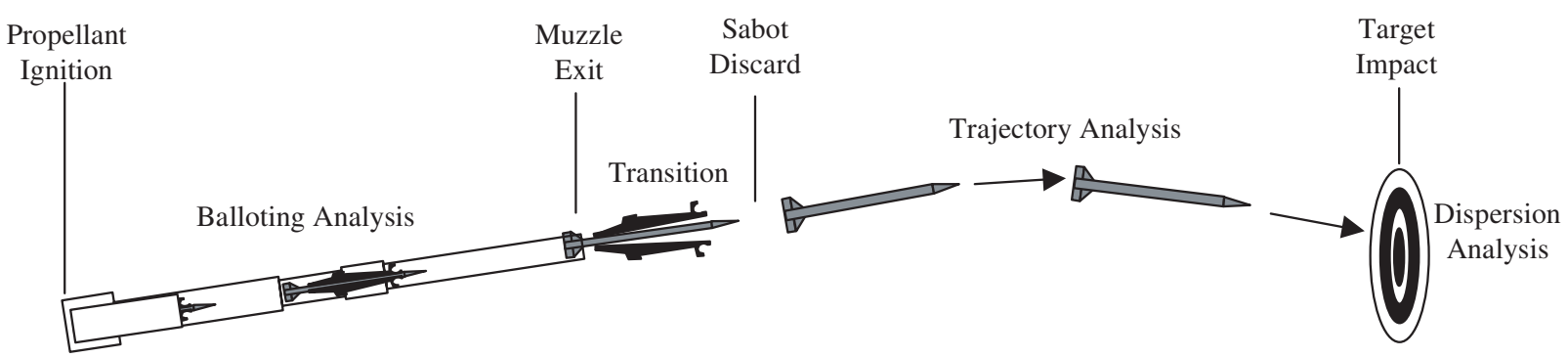

Fig. 1. The complete projectile environment.

tics. Since some of the inputs to this approach are statistical in nature, the historical data provides a foundation from which to derive the statistical information.

The last type of information required is test and/or measurement that is important to predicting dispersion but is not derived from analysis. This includes bore centerline measurements, bore site errors inherent within a test fixture or bore site tool, known sabot discard issues from tests of similar sabots, etc.

\subsection{System is based on PRODAS and BALANS}

PRODAS is a projectile design and analysis system with several analysis modules and is used to determine free-flight characteristics. The analysis modules that are key to this approach for predicting dispersion are the following:

- Model/Edit Module - The physical description of the projectile using PRODAS is used throughout the modeling effort. The lumped parameter model for in-bore balloting is automatically built from the geometric model created in this module.

- Physical Properties Module - This module uses the element / component definition from the Modeling Module to determine element, component and assembly physical properties that are used, not only in subsequent PRODAS analyses, but also in the in-bore balloting analysis.

- Stability Module - This module predicts the aerodynamic and stability coefficients for a given projectile configuration. Output from this module includes the Aerodynamic Jump Factor and the Aerodynamic Trim Coefficients.

- Interior Ballistics Module - This module's output is a tabulation of time, base pressure, chamber pressure, travel, spin rate, spin acceleration, axial acceleration, and axial velocity. These parameters form the forcing function for the in-bore balloting dynamic analysis.
- Trajectory Module - Both 4 DOF and 6 DOF trajectory options are used to assess the free-flight dispersion sensitivities.

- CG Offset Module - This module provides the ability to estimate the mean and standard deviation of projectile center of gravity radial offset and tilt of the principal axis from projectile drawings.

BALANS simulates the dynamic response and interaction of a flexible projectile and a flexible gun tube during in-bore travel. It also includes the effects of a curved bore profile. The simulation utilizes individual models of the projectile and gun tube, in a time step iterative solution. Pertinent motion and load data are periodically saved during the analysis to produce selective summary graphical displays. BALANS takes advantage of the Interior Ballistics simulation and CG Offset calculations of PRODAS and an automatic lumped parameter modeling capability to assist in building a BALANS model.

The analytical procedure utilized in BALANS presupposes that the projectile is initially misaligned within the gun tube due to manufacturing tolerances. During firing, this misalignment produces secondary forces causing transverse displacement and yawing motion of the projectile as it travels from breech to muzzle. The resulting yaw angle, angular rate, and transverse velocity at muzzle exit are then analyzed for their effect on dispersion.

\subsection{Propellant ignition to muzzle exit: in-bore dispersion components}

Balloting, within the context of this paper, refers to any transverse motion of a projectile in the gun tube. Some of the causes of balloting are discussed below.

- Projectile center of gravity (CG) and principal axis may be offset from the bore centerline. Even with a perfectly concentric projectile, it is possible to insert a projectile into a gun tube with less than 
perfect alignment with the bore centerline. Even if perfect insertion were achievable, projectile wear during travel may not be uniform, thereby producing CG or principal axis misalignment with the bore centerline.

- Projectiles, as manufactured, are not perfectly concentric. This lack of concentricity produces a transverse separation between the projectile geometric centerline (referenced from the bourrelet diameter) and the projectile CG.

- Projectile/gun tube (and wear induced) clearances may exist at projectile surfaces. These clearances enable the projectile to assume a skewed and/or non-symmetric position (CG shifted offaxis) within the gun bore.

- Insufficient stiffness of the projectile / gun tube interface will exacerbate conditions caused by minimal projectile misalignment. When a projectile is subjected to lateral loads, the radial stiffness of the projectile at the bourrelet, limits the radial deflection of the $\mathrm{CG}$ and potential tilt of the principal axis. Excessive deflection produces CG offsets that contribute to further deflection and balloting.

- Projectile wear produces or widens gaps (clearances) between the projectile bourrelet surfaces and the gun bore. Wear, therefore, enables the projectile to tilt its principal axis and/or translate the CG off the bore centerline.

- Gun tube curvature (including bore irregularities) exists in all gun tubes. While gun tube profiles are monitored during manufacture, some local tube curvature exists. Any tube curvature will induce balloting.

- Gas dynamics produce irregular pressures at the projectile base. These pressure spikes tend to exacerbate any existing projectile misalignment with the gun tube.

The basic inputs for the in-bore balloting analysis are a lumped parameter model of the projectile that properly characterizes its mass properties and flexibility, a forcing function, and several diameters and runouts that are used to orient the projectile within the gun tube. The lumped parameter model is generated automatically from the PRODAS geometric model.

Figure 2 is the representation of the stiffness (bending) model with lumped mass node locations of the $120 \mathrm{~mm}$ M865 APFSDS projectile that was automatically generated from the PRODAS geometric model; also shown is a lumped parameter model of a $120 \mathrm{~mm}$ gun tube. As shown, the upper half of the model is the actual projectile as generated from PRODAS. The lower half attempts to mirror the upper half by reflecting the lumped parameter node/element model. Although it is modeled and displayed as axisymmetric, it is handled as three-dimensional from a computational or analytical point of view.

The forcing function required for the balloting analysis is provided directly from the PRODAS interior ballistics analysis module. PRODAS uses either the BaerFrankl methodology of IBHVG2 to simulate propellant combustion and calculate the time-dependent parameters of base pressure to be applied to the projectile aft of the obturator during the balloting analysis. Spin velocity and acceleration are computed and used to calculate centrifugal forces during in-bore travel; axial velocity and acceleration are computed and used to calculate axial forces during in-bore travel. Transverse forces are calculated from the induced balloting motion.

The diameters and runouts that are used to orient the projectile within the gun tube are shown in Fig. 3. These define the locations of the projectile / gun tube interfaces and some of the critical projectile dimensions which effect dispersion. The runouts and tolerances for these dimensions are also required.

The statistical in-bore balloting analysis uses these dimensions described above and their tolerances to randomly orient the projectile in the gun tube. Several hundred in-bore balloting analyses are generally required to obtain statistically valid muzzle exit yaw, yaw rate, and transverse velocity predictions. To further illustrate this, the in-bore balloting analysis sequence number versus the cumulative average standard deviation of yaw rate is plotted in Fig. 4. Each sequence number represents a different in-bore balloting analysis with different projectile dimensions.

\subsection{Muzzle exit to sabot discard: transition dispersion components}

Transition sensitivities are separated into sabot discard and bore site sensitivities. Errors induced by sabot discard may have significant variation from one projectile configuration to another. There is both a physical component that can occur due to asymmetric loads applied to the core during discard and an aerodynamic interference component as shown in Fig. 5.

Sabot discard is the least well understood of the major contributors to dispersion and therefore is generally determined from test, observation, and/or experience. Experience has shown that sabot discard effects on dispersion can be minimized by ensuring that sabot discard is symmetric and that it happens quickly; sabots 


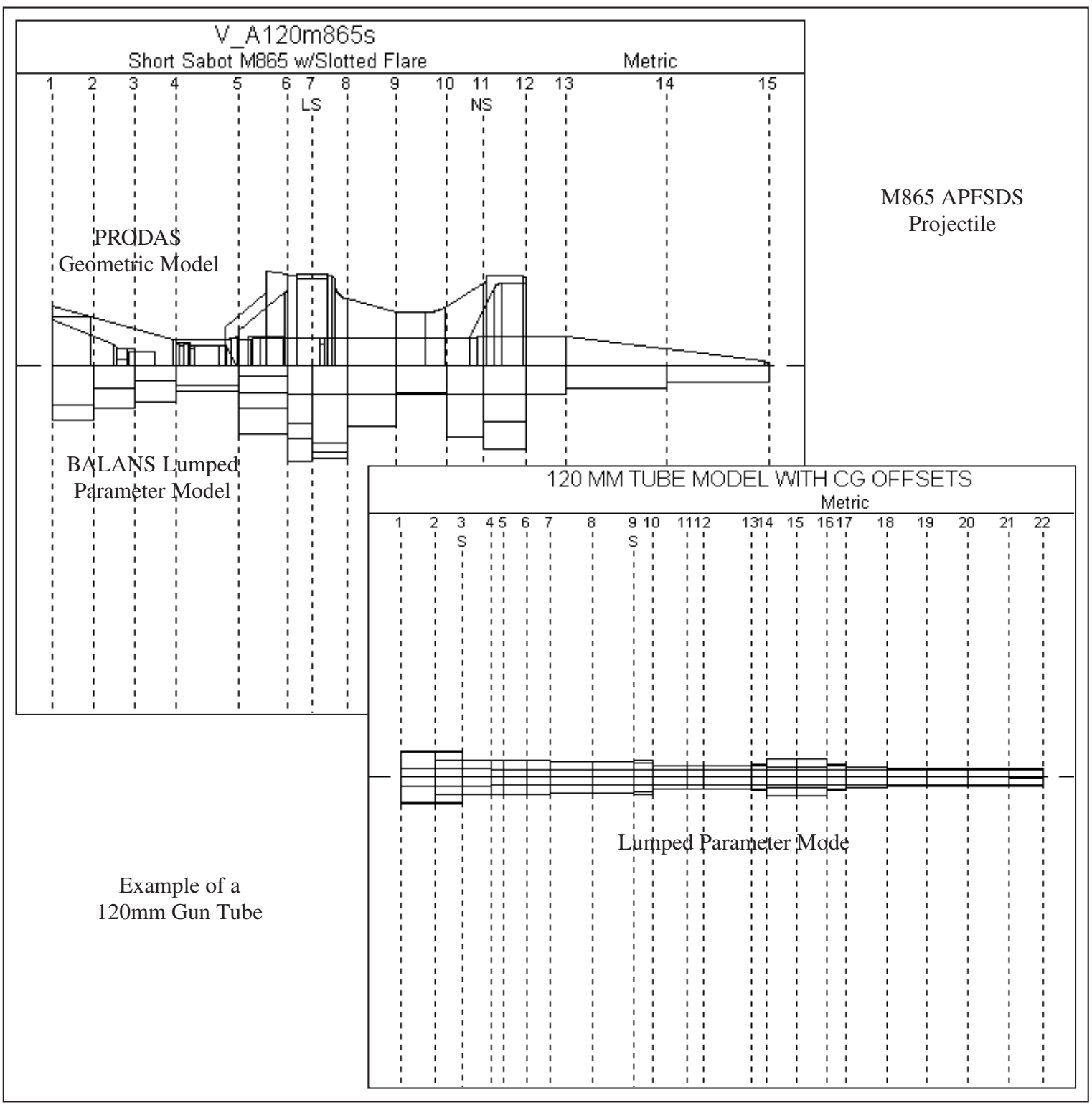

Fig. 2. Lumped parameter models of the projectile and the gun tube.

that tend to hang on too long often have poor dispersion. Other sabot design considerations to minimize the effect of sabot discard on dispersion include ensuring that the sabot pivot point is as close to the projectile center of pressure as possible and that the aft end of the sabot is designed to prevent gas leakage.

\subsection{Sabot discard to target impact: free-flight dispersion components}

The free-flight dispersion component sensitivities include muzzle velocity, aerodynamic jump, cross winds, aerodynamic trim angle, and aerodynamic/mass asymmetries. All of these parameters are determined via trajectory analysis within PRODAS as follows:

- The muzzle velocity sensitivity factor is the drop variation due to muzzle velocity variation and can be calculated by comparing the drop of trajectory simulations made by perturbating muzzle velocities (typically $10 \mathrm{M} / \mathrm{S}$ ). The resulting "delta drop" at the range of interest is then divided by the muzzle velocity difference to obtain the factor. Variations in muzzle velocity add primarily to the ver- 


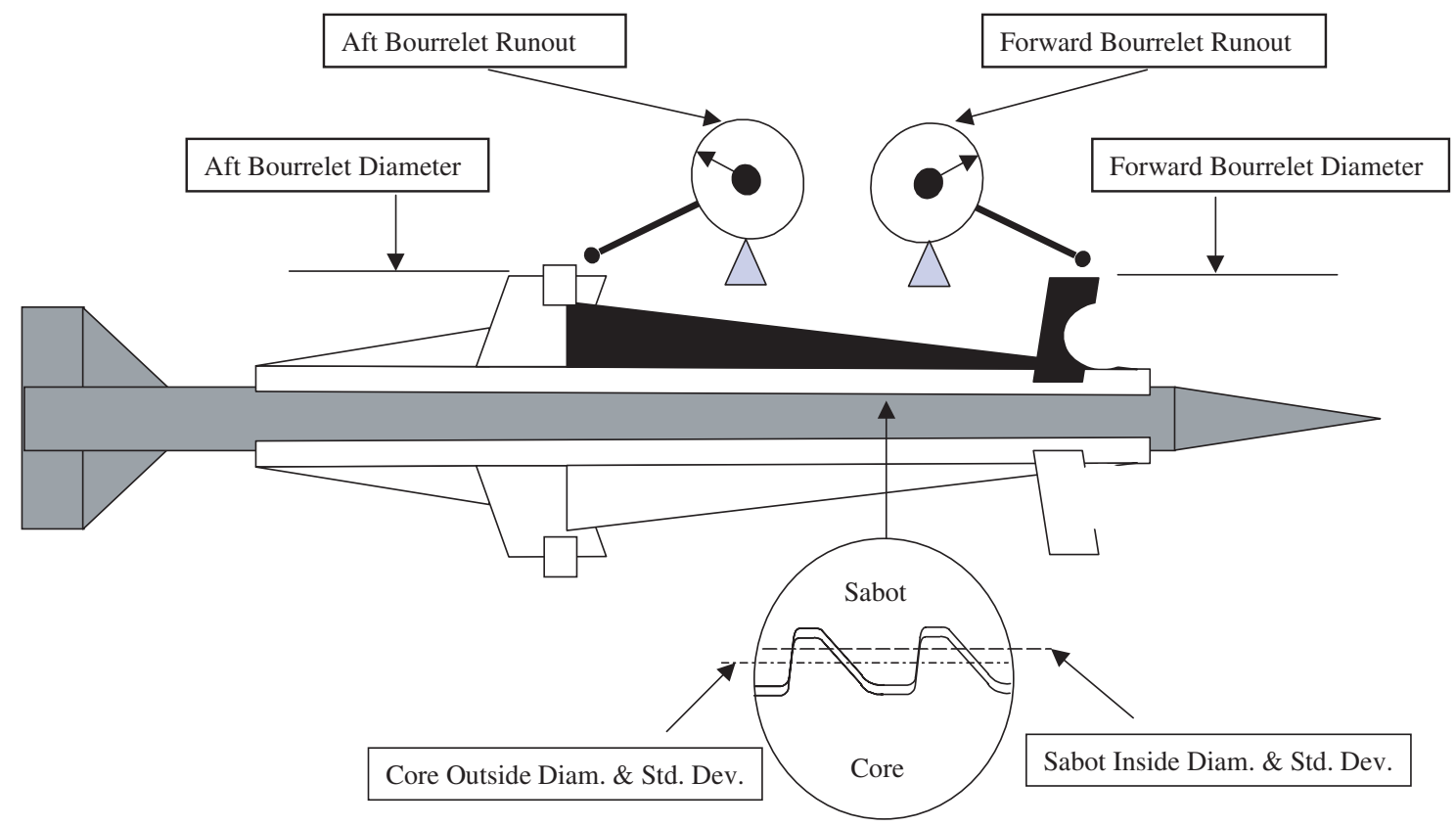

Fig. 3. Balloting analysis variables.

\section{Cumulative Standard Deviation of Yaw Rate (with Running Average)}

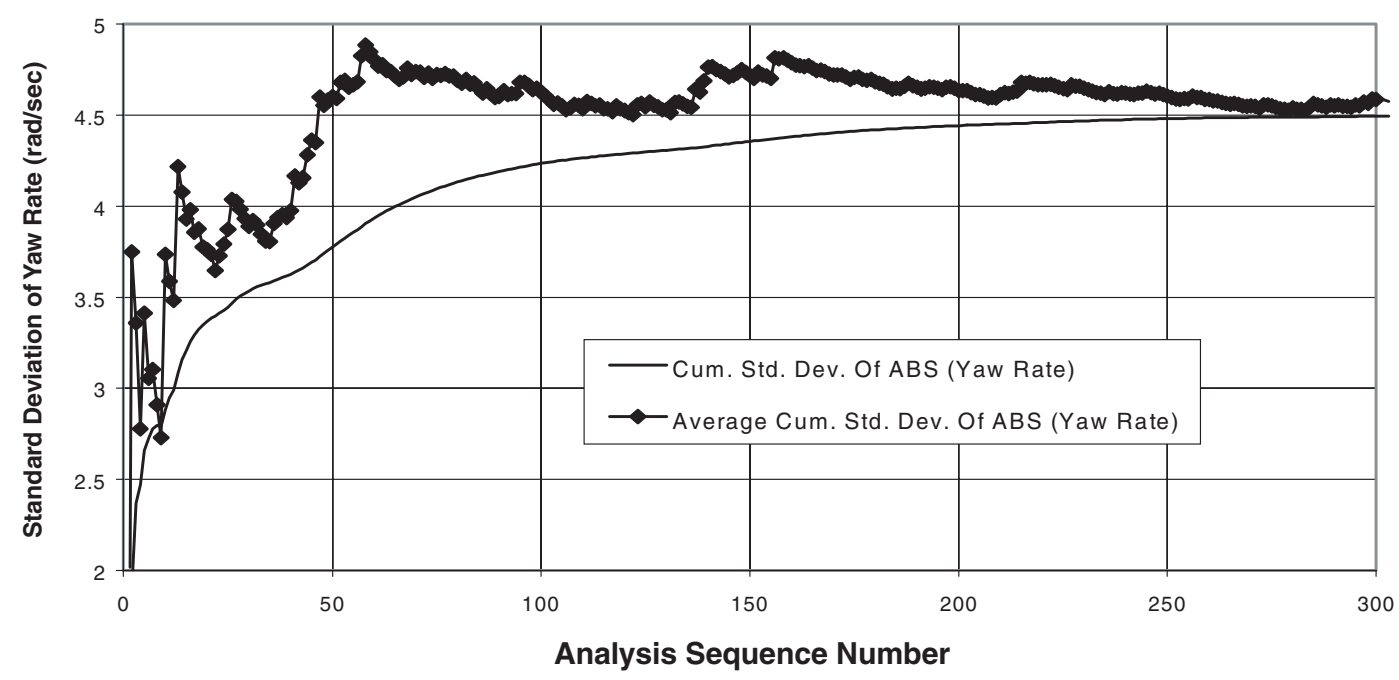

Fig. 4. Analysis sequence No. vs. cumulative standard deviation of yaw rate.

tical projectile dispersion. Muzzle velocity standard deviation, which is also required, is generally available for ammunition lots.

- The aerodynamic jump sensitivity relates dispersion to the muzzle exit yaw rate of the projectile. It may be calculated using PRODAS. This factor is dependent upon the physical and aerodynamic characteristics of the projectile as well as the projectile spin and velocity.

- Typically, the crosswind sensitivity of the projectile is determined by trajectory simulations of the projectile flight to the range of interest both with and without a nominal crosswind applied $(5 \mathrm{~m} / \mathrm{s})$. The wind sensitivity factor is computed by di- 


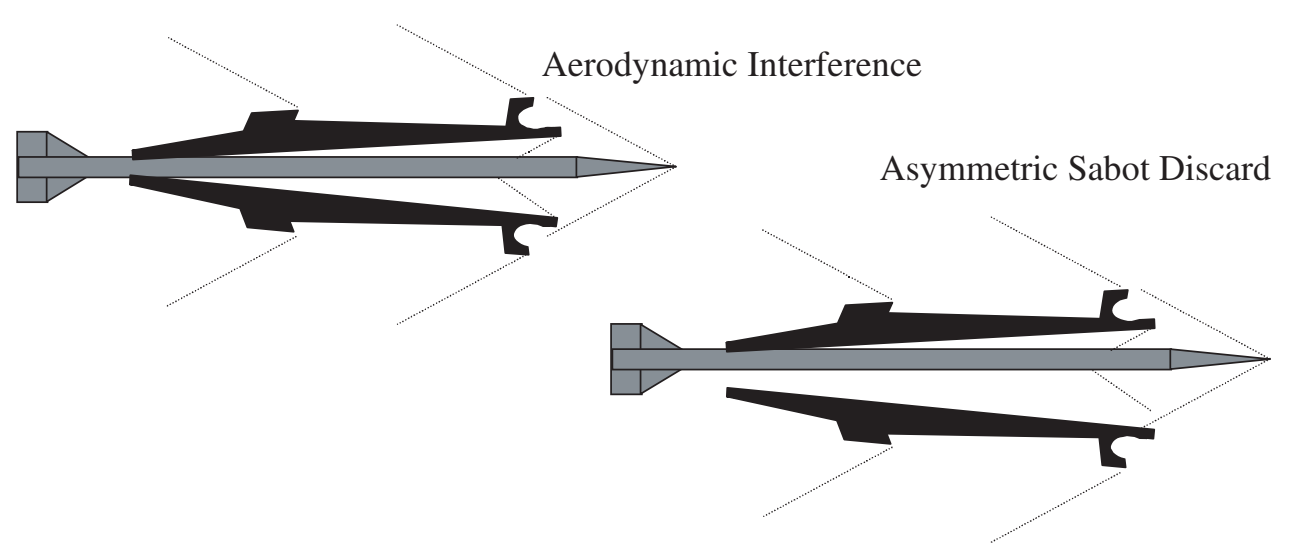

Fig. 5. Sabot discard components of dispersion.

viding the change in drift between the two cases (in mils) by the applied wind velocity. Cross wind standard deviation of the variation of the true wind velocity from the measured value (from range MET data).

- The aerodynamic trim angle of a projectile configuration (due to manufacturing tolerances) may be calculated from PRODAS predictions of the body alone and fin alone center of pressure and normal force coefficients, and from the expected one sigma value of the angular misalignments of the nose and tail sections. The calculated angle of the projectile geometric principal axis with the air stream flow assuming no spin. (This is analogous to the angle a projectile would take if suspended in a wind tunnel by its center of gravity.)

- The aerodynamic/mass asymmetry factor is determined by simulating trajectories with a trim angle assumed to be oriented at orthogonal and diametrically opposed orientations. This will produce an impact pattern at a specified range that can be fitted with a circle whose radius (in mils) can then be divided by the assumed trim angle to calculate the factor.

\subsection{Sabot front borerider stiffness vs. dispersion}

Total dispersion is computed by performing a rootmean-square combination of the following dispersion components:

- Propellant Ignition to Muzzle Exit (from In-Bore Balloting)

* Yaw

* Yaw Rate

* Transverse Velocity
* Muzzle Spin (combination of yaw, aerodynamic jump, spin rate)

- Muzzle Exit to Sabot Discard (from Experience/Experimental Results)

* Sabot Discard

* Bore Site

- Sabot Discard to Target Impact (from Free-Flight Trajectory Analysis)

* Muzzle Velocity

* Wind Component

* Aerodynamic and Mass Asymmetries

The in-bore balloting analysis is automatically performed for a range of theoretical stiffness values to arrive at a curve of stiffness vs. dispersion as shown in Fig. 6. For each stiffness value, several hundred analyses, each with its own randomized initial conditions, were run to arrive at a single dispersion value for that stiffness. Over the range of stiffness values selected, the example analysis shows that the dispersion decreases dramatically for stiffness values less than $300000 \mathrm{lb} / \mathrm{in}$; beyond this point, increasing stiffness values do not reduce dispersion thereby defining this point as the knee in the curve. Dispersion components from muzzle exit to sabot discard and sabot discard to target impact are held constant for this analysis since they are not a function of the sabot front borerider stiffness.

\section{Sample results: dispersion vs. stiffness in the $120 \mathrm{~mm}$ m865}

The $120 \mathrm{~mm}$ M865 APFSDS production round was selected to illustrate this analysis process. In addition 


\section{Dispersion vs. Radial Stiffness Example}

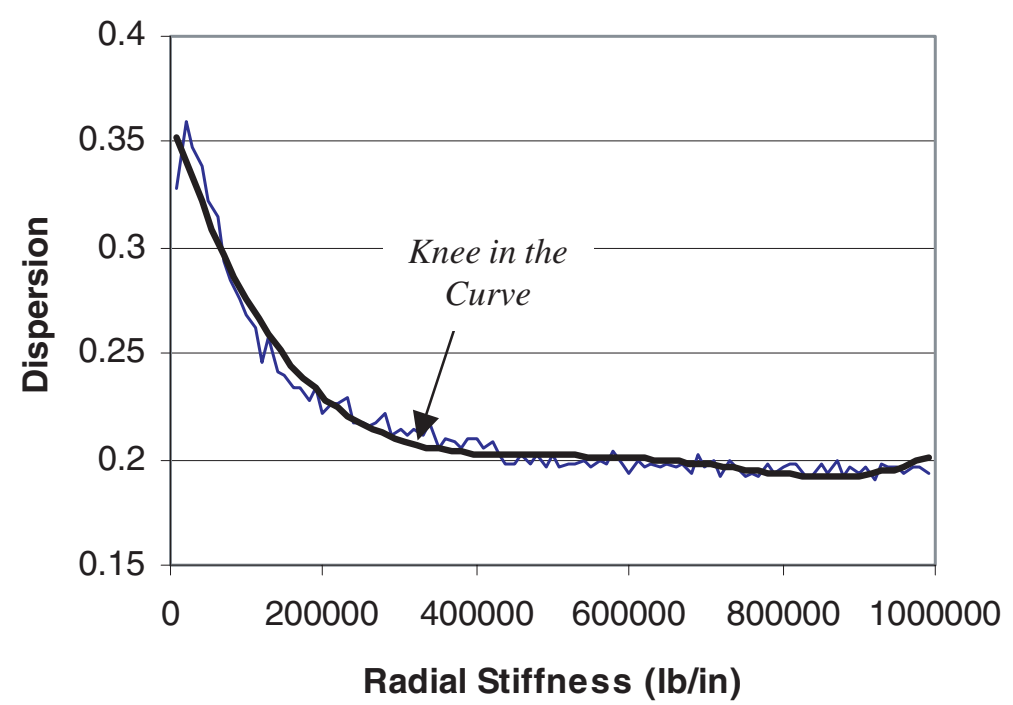

Fig. 6. Example of sabot front borerider stiffness vs. total dispersion.

M865: Dispersion vs. Radial Stiffness

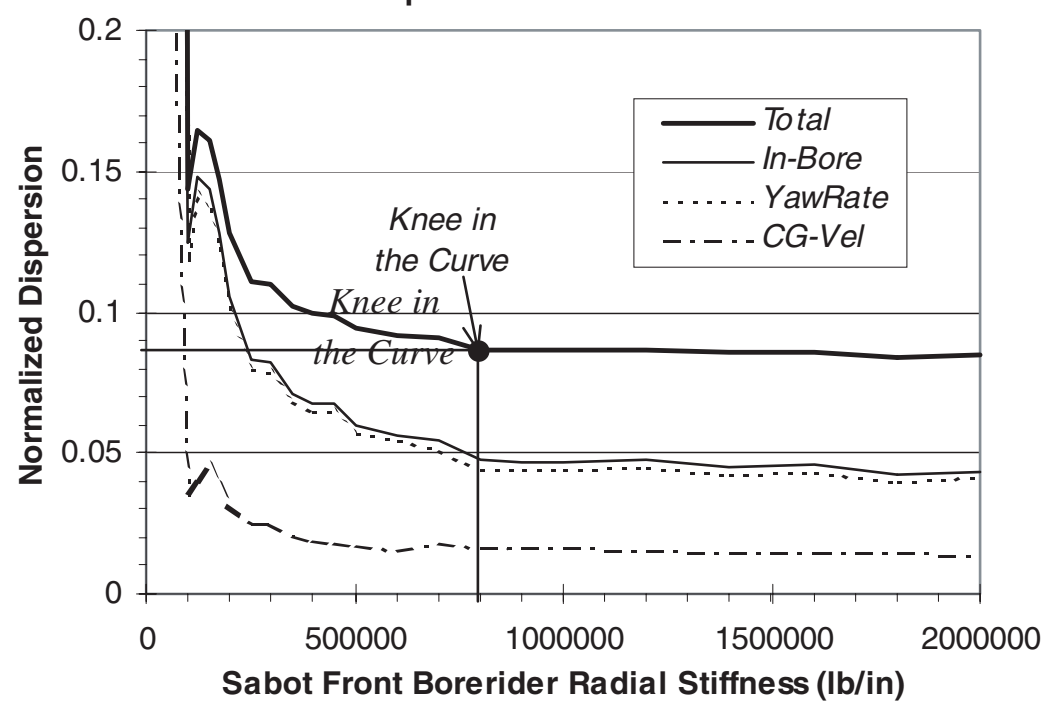

Fig. 7. M865 test case, radial stiffness vs. normalized dispersion.

to the technical data package, statistical process control (SPC) data was collected to develop the necessary manufacturing tolerances of the diameters and runouts required by this analysis system.

Figure 7 is a plot of the sabot front borerider stiffness vs. dispersion (dispersion values have been normalized) for the M865 projectile. From testing, the normalized dispersion value for the M865 is approximately 0.085 . It is obvious that a radial stiffness above
$800000 \mathrm{lb}$./in provides little or no improvement in dispersion. This point will vary depending on the projectile/sabot configuration.

\section{Summary and conclusions}

The analysis process described in this paper can not only determine the effect of a change in front borerider 
120mm M865 APFSDS

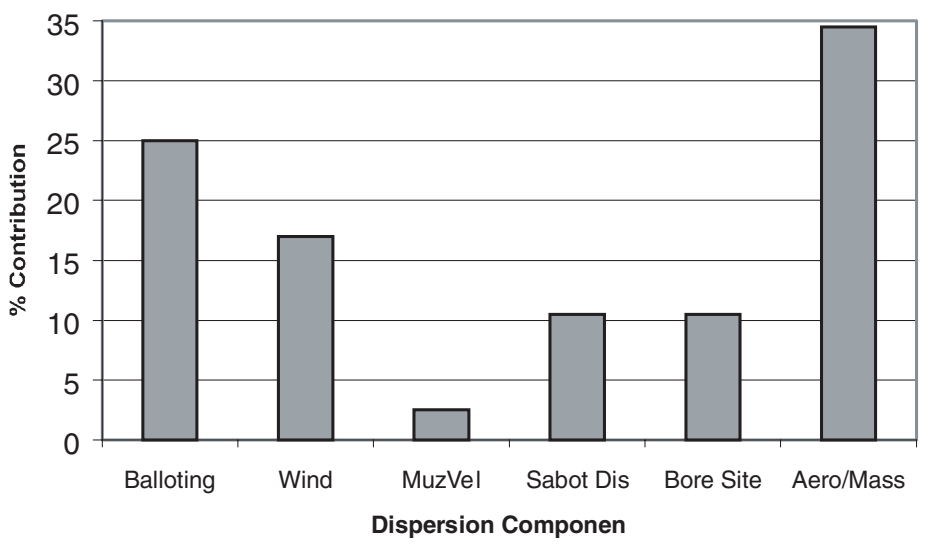

Fig. 8. M865 dispersion components.

stiffness but it can also identify the components of dispersion and their relative contribution as shown in Fig. 8. This can greatly assist the projectile designer in choosing a dispersion source to attack in trying to improve dispersion.

Following are some conclusions based on the use of this system for predicting dispersion and solving dispersion-related problems on several projectiles:

- This approach enables the characterization of the components of dispersion.

- Use of this system can lead to cost effective test plans by giving the engineer the relative magnitude of dispersion improvement to be expected by changes in configuration.

- This approach provides realistic expectations for a projectile design's dispersion capability.

- Use of this system has proven to be very useful when coupled with data from spark range testing that can provide accurate aerodynamic coefficients and average trim estimates.

- The system is useful in the investigation of piece part dimensional tolerances and their effect on dispersion.

- The system provides insight into which parameters need to be modified to enhance projectile dispersion and/or minimize external effects (i.e., wind) while testing for dispersion.

\section{References}

[1] A.F. Hathaway and J.R. Burnett, Stochastic Approach to Predicting Dispersion, Proceedings, 49th Aeroballistic Range Association Meeting, The Hague, Netherlands, October, 1998.

[2] B.K. Stearns and J.R. Burnett, Simulation of Projectile Balloting in Hypervelocity Launchers, Proceedings, 45th Aeroballistic Range Association Meeting, Huntsville, Alabama, October, 1994.

[3] R.H. Whyte, B.K. Stearns, J.R. Burnett and J.A. Siewert, M865 Short Sabot and M831 Dispersion Sensitivity Analysis, Internal Report, Arrow Tech Associates, South Burlington, Vermont, September 1990.

[4] J.A. Siewert, B.K. Stearns and J.R. Burnett, Aluminum and Plastic M865 Variants, Aeroballistic Analysis, Dispersion Prediction, and Balloting Response Analysis, Internal Report, Arrow Tech Associates, South Burlington, Vermont, April 1994.

[5] D.H. Lyons, Radial Stiffness Measurements of 120MM Projectiles, Aberdeen Proving Ground, Proceedings of the Seventh U.S. Army Symposium on Gun Dynamics, ARCCB-SP-93034 Newport, R.I., Sponsored by: Benet Laboratories, Watervliet, N.Y. 11-13 May, 1993, pp. 167-186.

[6] T.E. Simkins, Transverse Response of Gun Tubes to CurvatureInduced Load Functions, Proceedings of the Second U.S. Army Symposium on Gun Dynamics, ARLCB-SP-78013, Renselaerville, N.Y., Sponsored by: Benet Laboratories, Watervliet, N.Y., September 1982, pp. I-67-77.

[7] Arrow Tech Associates, PRODAS Version 3.9 Users and Technical Manual, Arrow Tech Associates, South Burlington, Vermont, January 1998.

[8] Arrow Tech Associates, BALANS Version 2.00 Users and Technical Manual, Arrow Tech Associates, South Burlington, Vermont, August 1998. 

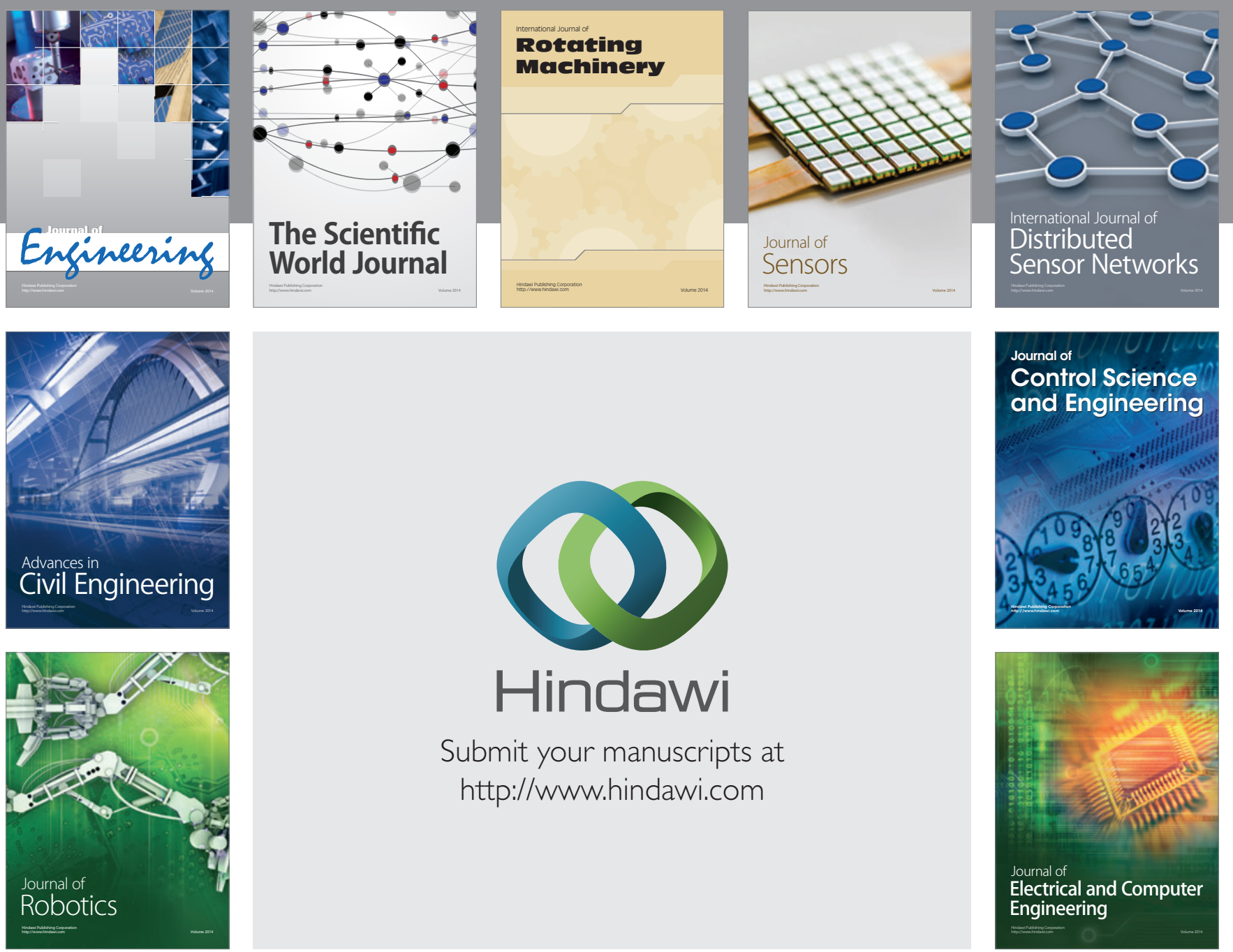

Submit your manuscripts at

http://www.hindawi.com
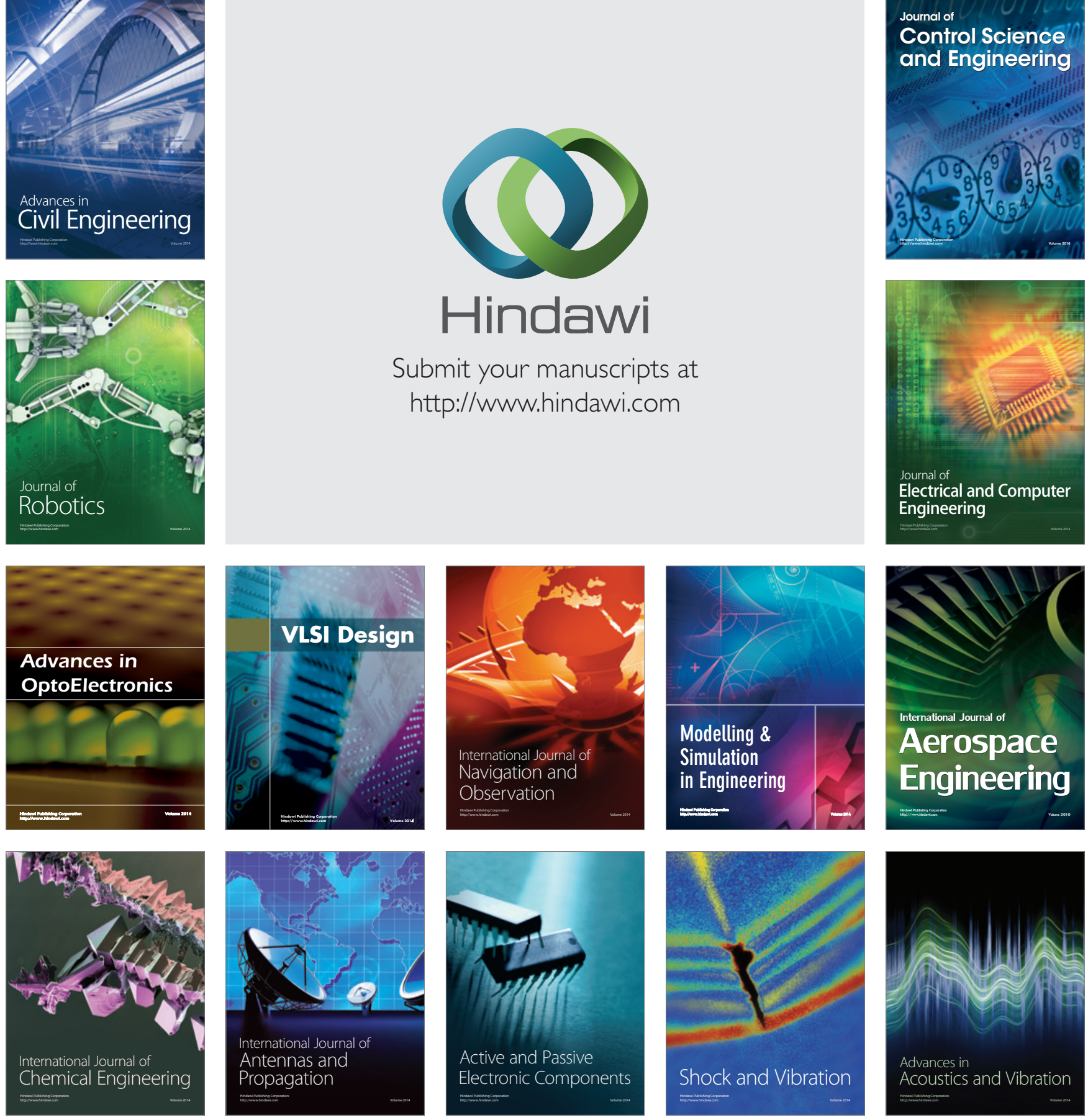